\title{
Intravenous Thrombolysis Prior to Mechanical Thrombectomy in Acute Ischemic Stroke: Silver Bullet or Useless Bystander?
}

\author{
Federico Di Maria, ${ }^{\text {a }}$ Mikael Mazighi, ${ }^{\text {bc }}$ Maéva Kyheng, ${ }^{\mathrm{c}}$ Julien Labreuche, ${ }^{\mathrm{c}}$ Georges Rodesch, ${ }^{\mathrm{a}}$ \\ Arturo Consoli, ${ }^{a}$ Oguzhan Coskun, ${ }^{a}$ Benjamin Gory, ${ }^{\mathrm{d}, \mathrm{e}}$ Bertrand Lapergue, ${ }^{\mathrm{f}}$ on behalf of the ETIS \\ (Endovascular Treatment in Ischemic Stroke) Study Group \\ aDepartment of Diagnostic and Therapeutic Neuroradiology, Foch Hospital, Suresnes, France

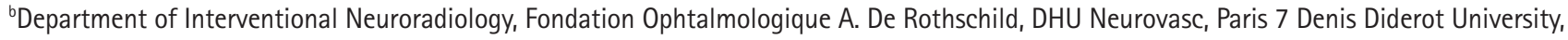 \\ Paris, France \\ 'University of Lille, CHU Lille, Lille, France

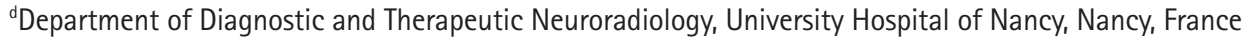 \\ eUniversity of Lorraine, Nancy, France \\ fDepartment of Neurology, Foch Hospital, Suresnes, France
}

Background and Purpose Recent single-center series and meta-analyses suggest that mechanical thrombectomy (MT) without prior intravenous thrombolysis (IVT) might be equally effective to bridging therapy. We analyzed, within the Endovascular Treatment in Ischemic Stroke (ETIS) prospective observational registry, the angiographic and clinical outcomes after IVT+MT versus MT alone.

Methods From December 2012 to December 2016, a total of 1,507 consecutive patients with a proximal arterial occlusion of the anterior circulation were treated by MT. Of these, 975 (64.7\%) received prior IVT. Immediate angiographic and clinical outcomes at 90 days (modified Rankin Scale [mRS]) were compared between the two groups while checking for propensity score, matchedpropensity score and by inverse probability of treatment weighting (IPTW) propensity score method. Results Favorable outcome (mRS 0 to 2 ) was more frequently achieved after IVT+MT ( $n=523$, $53.6 \%)$ than after MT alone $(n=222,41.8 \%)$ with an unadjusted odds ratio (OR) for bridging therapy of 1.61 (95\% confidence interval $[\mathrm{Cl}], 1.29$ to 2.01). This difference remained not significant in matched-propensity score cohort $(\mathrm{OR}, 1.21 ; 95 \% \mathrm{Cl}, 0.90$ to 1.63$)$ although it remained according to adjusted propensity score $(\mathrm{OR}, 1.31 ; 95 \% \mathrm{Cl}, 1.02$ to 1.68$)$ and IPTW (OR, $1.37 ; 95 \% \mathrm{Cl}, 1.09$ to 1.73 ) analyses. A significant difference was found in terms of excellent outcome ( $m R S O$ to 1) (adjusted $\mathrm{OR}, 1.63 ; 95 \% \mathrm{Cl}, 1.25$ to 2.11) and successful reperfusion (adjusted $\mathrm{OR}, 1.58 ; 95 \% \mathrm{Cl}, 1.33$ to 2.15 ). No differences in intracerebral hemorrhage or in allcause mortality within 90 days were found between groups.

Conclusions IVT prior to MT is associated with increased excellent outcome and successful reperfusion rates. These findings support the use of bridging therapy.

Keywords Ischemic stroke; Intravenous thrombolysis; Tissue plasminogen activator; Thrombectomy
Correspondence: Federico Di Maria Department of Diagnostic and Therapeutic Neuroradiology, Foch Hospital, 40 Rue Worth, 92151 Suresnes, France

Tel: $+33-146252526$

Fax: +33-146252132

E-mail: federico.dimaria@gmail.com

Received: May 25, 2018

Revised: September 8, 2018

Accepted: September 10, 2018 


\section{Introduction}

After the overwhelming results from randomized controlled trials establishing the safety and effectiveness of endovascular thrombectomy (ET) in the setting of acute ischemic stroke (AIS) for patients with emergent large vessel occlusion (ELVO) within the anterior circulation, ${ }^{1-3}$ questions have been raised concerning the relevance of giving intravenous thrombolysis (IVT) prior to ET. Current recommendations by the American Heart Association/ American Stroke Association ${ }^{4}$ and European Stroke Organisation ${ }^{5}$ advocate that IVT should be offered to all eligible patients in first line, based on the fact that more than $80 \%$ of them were treated with IVT in the above cited trials." However, more recent observational data from single-center series, as well as pooled analyses and meta-analyses from the published studies, suggest that direct ET without IVT might be equally effective to bridging therapy (i.e., IVT prior to ET) ${ }^{6-10}$ Additionally, concerns exist about potential side effects of IV tissue-plasminogen activator (tPA) in patients with ELVO undergoing ET, including increased intracerebral hemorrhage $(\mathrm{ICH})$ risks, responsible for limitations in the use of antithrombotic therapy (e.g., antiplatelets and heparin). ${ }^{6}$ Prior IVT may also complexify AIS patient workflow, particularly in local stroke centers which apply a "drip and ship protocol," thereby causing further delays in ET initiation."

In this study, we investigated in the real-world observational Endovascular Treatment in Ischemic Stroke (ETIS) registry the impact of the two therapeutic strategies IVT+mechanical thrombectomy (MT) versus MT alone on angiographic and functional outcomes.

\section{Methods}

\section{Patients and variables}

Based on local regulations, neither patient informed consent nor approval by the Institutional Ethics Committee was required given the retrospective design of this study. From December 2012 to December 2016, we collected clinical and imaging data from three different comprehensive stroke centers taking part in the ETIS registry. ${ }^{12}$ Prospectively gathered data was then retrospectively analysed.

Patients included in our study presented with an AIS within six hours of stroke onset, and imaging evidence of anterior circulation occlusion. Age, baseline modified Rankin Scale (mRS) score, low or high National Institutes of Health Stroke Scale (NIHSS) and infarct core volume were not considered as exclusion criteria per se. Middle cerebral artery (MCA) occlusions were divided into proximal (M1) and distal (M2). Carotid occlusions were divided into terminal intracranial (carotid T), and tandem (extracranial internal carotid artery occlusion associated with an intracranial artery occlusion). A stroke neurologist decided whether or not to perform IVT, based on conventional inclusion and exclusion criteria for IVT, according to United States and European Union guidelines. ${ }^{4,5}$

Contraindications for IVT were: late treatment (between 4.5 and 6 hours after onset) and treatment in patients with a possible high bleeding risk after thrombolytic therapy, including elevated increased international normalized ratio (1.7 to 3.0), thrombocyte count below $90 \times 10^{9} / \mathrm{L}$, history of $\mathrm{ICH}$, severe head injury in the preceding 4 weeks, previous AIS in the preceding 6 weeks and major surgery, gastrointestinal bleeding or urinary tract bleeding within the previous 2 weeks.

All endovascular procedures were performed under a dedicated neuroanesthesic protocol that encompassed conscious sedation or general anesthesia. All procedures were performed using either an aspiration catheter, a stent retriever or both, at the operator's discretion.

\section{Outcomes}

The primary study outcome was the percentage of patients who achieved a favorable 90-day outcome, defined as a mRS score of 0 to 2 or equal to pre-stroke Rankin score. Secondary outcomes included clinical outcomes (excellent 90-day outcome defined as a mRS score of 0 to 1 or equal to pre-stroke Rankin score, 90-day all-cause mortality, any hemorrhagic complications and symptomatic intracerebral hemorrhage $[\mathrm{sICH}]$ defined as a hemorrhage on the follow-up computed tomography/magnetic resonance imaging scan associated with an increase of 4 points in NIHSS score) and procedural outcomes (successful reperfusion defined by modified Thrombolysis In Cerebral Infarction [mTICl] 2b/3, excellent reperfusion defined by $\mathrm{mTICl} 3$, rescue therapy, more than two passes, time from groin puncture to successful reperfusion, emboli to uninvolved territory, vessel perforation and vasospasm).

\section{Statistical analysis}

Continuous variables are expressed as mean \pm standard deviation (SD) in the case of normal distribution or medians (interquartile range) otherwise. Categorical variables are expressed as numbers (percentage). Normality of distributions was assessed using histograms and the Shapiro-Wilk test.

We assessed the effect of the therapeutic approach on clinical outcomes using logistic regression models and calculated the odds ratio (OR) for the MT+IVT relative to the MT group as the treatment effect size. In order to reduce the effects of potential confounding factors in the between-group comparisons, we used propensity-score methods. ${ }^{13-16}$ As the main analysis, 
propensity score was used to assemble well-balanced groups (propensity score-matched cohort) and a generalized linear mixed model was used to take into account the matched design. As secondary analyses, the effect of the therapeutic approach were estimated using propensity score adjustment method (propensity score considered as a covariate in a multivariable logistic regression model) and inverse probability of treatment weighting (IPTW) propensity score method (using stabilized inverse propensity score as weight in simple logistic regression model) were performed. The propensity score was

Table 1. Baseline characteristics according to use of intravenous thrombolysis prior to endovascular treatment before and after propensity score-matching

\begin{tabular}{|c|c|c|c|c|c|c|}
\hline \multirow[b]{2}{*}{ Characteristic } & \multicolumn{3}{|c|}{ Before propensity score-matching } & \multicolumn{3}{|c|}{ After propensity score-matching* } \\
\hline & $\begin{array}{c}\text { MT } \\
(n=531)\end{array}$ & $\begin{array}{l}\text { MT+IVT } \\
(n=976)\end{array}$ & $\begin{array}{l}\text { ASD } \\
(\%)\end{array}$ & $\begin{array}{c}\text { MT } \\
(n=407)\end{array}$ & $\begin{array}{l}\text { MT+IVT } \\
(n=407)\end{array}$ & $\begin{array}{l}\text { ASD } \\
(\%)\end{array}$ \\
\hline Age (yr) & $67.6 \pm 15.1$ & $67.2 \pm 15.0$ & 2.6 & $66.1 \pm 15.3$ & $66.9 \pm 15.5$ & 4.9 \\
\hline Male sex & $272(51.2)$ & $530(54.3)$ & 6.2 & $217(53.0)$ & $207(50.9)$ & 5.0 \\
\hline Direct admission & $161(30.3)$ & $299(30.6)$ & 0.7 & $123(30.2)$ & $112(27.5)$ & 6.1 \\
\hline \multicolumn{7}{|l|}{ Medical history } \\
\hline Hypertension & $311(58.6)$ & $511(52.4)$ & 12.9 & $218(53.6)$ & $230(56.5)$ & 6.0 \\
\hline Diabetes & $92(17.3)$ & $156(16.0)$ & 3.6 & $67(16.5)$ & $72(17.7)$ & 3.2 \\
\hline Hypercholesterolemia & $157(29.6)$ & $289(29.6)$ & 0.1 & $116(28.5)$ & $115(28.3)$ & 0.4 \\
\hline Current smoking & $144(27.1)$ & $230(23.6)$ & 8.5 & $111(27.3)$ & $111(27.3)$ & 0.03 \\
\hline Antiplatelet use & $137(25.8)$ & $269(27.6)$ & 3.5 & $114(28.0)$ & $113(27.8)$ & 0.9 \\
\hline Anticoagulations & $181(34.1)$ & $74(7.6)$ & 67.4 & 74 (18.2) & 74 (18.2) & 0.06 \\
\hline NIHSS score & $16(11-21)$ & $17(11-20)$ & 1.0 & $16(11-21)$ & $16.5(11-21)$ & 3.6 \\
\hline ASPECTS & $7(6-8)$ & $7(6-8)$ & 4.6 & $7(6-8)$ & $7(6-8)$ & 0.2 \\
\hline Pre-stroke rankin $\geq 1$ & $124(23.4)$ & $117(12.0)$ & 28.4 & $70(17.2)$ & 73 (17.9) & 1.8 \\
\hline Site of occlusion & & & & & & 5.6 \\
\hline M1-MCA & $269(50.7)$ & 499 (51.1) & 11.4 & $200(49.1)$ & $201(49.4)$ & \\
\hline M2-MCA & 65 (12.2) & $129(13.2)$ & & 46 (11.3) & 50 (12.3) & \\
\hline Carotid T & $113(21.3)$ & $168(17.2)$ & & 85 (20.9) & $80(19.6)$ & \\
\hline Tandem & $84(15.8)$ & $180(18.5)$ & & $76(18.7)$ & 76 (18.7) & \\
\hline Favorable collaterals & $291(54.8)$ & $596(61.1)$ & 16.6 & $233(57.2)$ & $237(58.2)$ & 2.1 \\
\hline \multicolumn{7}{|l|}{ Stroke etiology } \\
\hline Large-artery atherosclerosis & $74(14.0)$ & $171(17.5)$ & 14.6 & $69(17.0)$ & $71(17.4)$ & 5.4 \\
\hline Cardioembolic & $303(57.0)$ & $490(50.2)$ & & $205(50.3)$ & $210(51.6)$ & \\
\hline Others & $154(29.0)$ & $315(32.3)$ & & $133(32.7)$ & $126(31.0)$ & \\
\hline \multicolumn{7}{|l|}{ EVT characteristics } \\
\hline Onset to groin puncture (min) & $244(185-308)$ & $235(190-285)$ & 19.6 & $244(186-300)$ & $250(200-303)$ & 2.3 \\
\hline Onset to imaging & 119 (85-174) & $110(86-144)$ & 29.7 & 119 (85-169) & $121(91-162)$ & 2.1 \\
\hline Imaging to groin puncture & $108(67-152)$ & $121(84-154)$ & 4.8 & $109(68-152)$ & $125(88-156)$ & 5.3 \\
\hline General anesthesia & $161(30.3)$ & $218(22.3)$ & 18.2 & $123(30.2)$ & $117(28.7)$ & 3.4 \\
\hline \multicolumn{7}{|l|}{ First-line strategy } \\
\hline ADAPT & $239(45.0)$ & $440(45.1)$ & 4.5 & $175(43.0)$ & $192(47.2)$ & 10.0 \\
\hline Stent retriever & $229(43.2)$ & $418(42.8)$ & & $180(44.2)$ & $165(40.5)$ & \\
\hline ADAPT+Stent retriever & $38(7.1)$ & $76(7.8)$ & & $33(8.1)$ & $30(7.4)$ & \\
\hline Other & $25(4.7)$ & $42(4.3)$ & & $19(4.7)$ & $20(4.9)$ & \\
\hline
\end{tabular}

Values are presented as mean \pm standard deviation, number $(\%)$, or median (interquartile range). Values were calculated after handing missing data using multiple imputation procedure.

MT, mechanical thrombectomy; IVT, intravenous thrombolysis; ASD, absolute standardized difference; NIHSS, National Institutes of Health Stroke Scale; ASPECTS, Alberta Stroke Program Early CT Score; MCA, middle cerebral artery; EVT, endovascular treatment; ADAP, a direct aspiration first pass technique. *Propensity score was calculated with all parameters in this Table 1. 
estimated using a non-parsimonious multivariable logistic regression model, with the treatment group as the dependent variable and all of the characteristics listed in Table 1 as covariates. Patients from the MT+IVT group were matched 1:1 to patients in the MT group according to propensity score using the greedy nearest neighbor matching algorithm with a caliper width of 0.2 SD of logit of propensity score. ${ }^{14,16}$ To evaluate bias reduction using the propensity score methods, absolute standardized differences (ASDs) were calculated. ${ }^{16}$ Because of missing baseline data (Supplementary Table1), we estimated the treatment effect size in propensity score-matched and -adjusted cohorts after handling missing covariate values by multiple imputation using a regression switching approach (chained equations with $m=10$ ). Imputation procedure was performed under the missing at random assumption ${ }^{17}$ using all variables listed in Table 1 (including treatment group) with a predictive mean matching method for continuous variables and multinomial or binary logistic regression model for categorical variables. In each imputed dataset, we calculated the propensity score and assembled a matched cohort to provide both adjusted and matched ORs. We therefore combined the ORs from each imputed dataset using Rubin's rules. ${ }^{18}$

Finally, we investigated the heterogeneity in treatment effect size for primary outcome across the following subgroups: age ( $<80$ years vs. $\geq 80$ years), admission NIHSS $(<10,10$ to 17 , $\geq 18$ ), Alberta Stroke Program Early CT Score (ASPECTS: $<5,5$ to $6, \geq 7$ ), site of occlusion (carotid terminus vs. tandem vs. M1MCA vs. M2-MCA), admission mode (mothership vs. drip and ship) and antiplatelet use (yes vs. no). Heterogeneity across subgroups was quantified by introducing a multiplicative term into logistic regression models. Statistical testing was conducted at the two-tailed a-level of 0.05. Data were analyzed using the SAS software version 9.4 (SAS Institute, Cary, NC, USA).

\section{Results}

\section{Population and participating centers}

A total number of 1,507 patients with an arterial occlusion in the anterior circulation were treated by MT at three comprehensive stroke centers. Of these, 975 (64.7\%) who received prior IVT and 407 matched pairs (mean value of the 10 imputed datasets) could be included (Supplementary Figure 1). Table 1 shows the baseline characteristics after handling missing values by multiple imputation according to the two study groups before and after propensity score-matching (Supplementary Table 1 for baseline characteristics before matching and handling missing values). Before matching, several meaningful differences (ASD >10\%) were found; as expected, the stronger difference was observed for anticoagulation therapy at stroke onset (34.1\% in MT vs. 7.5\% MT+IVT) which was a contraindication to use IVT. These differences were reduced after propensity score-methods with a maximum ASD of 10\% for first-line thrombectomy strategy in matched-propensity score (Table 1, Supplementary Table 2, and Supplementary Figure 2).

\section{Clinical outcomes}

Favorable outcome at 90 days (i.e., primary outcome) was achieved in $49.4 \%(n=745)$ of patients overall, with a significant difference between the two groups in favor of the IVT+MT approach, with an unadjusted OR of 1.61 (95\% confidence interval $[\mathrm{Cl}], 1.29$ to 2.01). However, this difference remained not significant in matched-propensity score cohort (OR, 1.21; $95 \%$ $\mathrm{Cl}, 0.90$ to 1.63$)$ although it remained when propensity score adjustment (OR, 1.31; 95\% Cl, 1.02 to 1.68) (Figure 1) or IPTW (OR, 1.37; 95\% Cl, 1.09 to 1.73) (Supplementary Table 3) method were used. Similar to favorable outcome, excellent outcome was achieved more frequently in MT+IVT than in MT group (Figure 1). This difference remained significant after propensity-score matching $(\mathrm{OR}, 1.45 ; 95 \% \mathrm{Cl}, 1.05$ to 2.01$)$, propensity score adjustement $(\mathrm{OR}, 1.63 ; 95 \% \mathrm{Cl}, 1.25$ to 2.11$)$, and IPTW (OR, 1.71; 95\% Cl, 1.33 to 2.21$)$ analyses.

Regarding safety outcomes, all-cause mortality within 90 days after symptom onset occurred less often in IVT+MT patients (17.6\%) than in MT patients (24.9\%; unadjusted OR, $0.64 ; 95 \% \mathrm{Cl}, 0.49$ to 0.84$)$. However, this difference did not remain significant regardless of the propensity score method used (Figure 1 and Supplementary Table 3). There was no difference between the two study groups regarding any $\mathrm{ICH}$ or sICH before and after using propensity score methods.

\section{Procedural outcomes}

Successful reperfusion was achieved more frequently in IVT+MT (79.1\%) than in MT patients (69.1\%; unadjusted OR, $1.69 ; 95 \% \mathrm{Cl}, 1.33$ to 2.15$)$. This difference in favor of the IVT+MT approach remained significant in propensity-score matched, adjusted and IPTW analyses (Figure 1 and Supplementary Table 3). However no such difference was observed with excellent reperfusion. Regarding other efficacy procedural outcomes, cases of emboli to uninvolved territory and of more than two thrombectomy device passes were less frequently observed in IVT+MT than in MT patients with an unadjusted $\mathrm{OR}$ of $0.66(95 \% \mathrm{Cl}, 0.44$ to 0.99$)$ and $0.74(95 \% \mathrm{Cl} 0.60$ to 0.93), respectively. Conversely, successful reperfusion was more often achieved within 45 minutes after groin puncture in the IVT+MT group than after MT alone, with an unadjusted OR of $1.32(95 \% \mathrm{Cl}, 1.06$ to 1.65$)$. These differences did not remain 


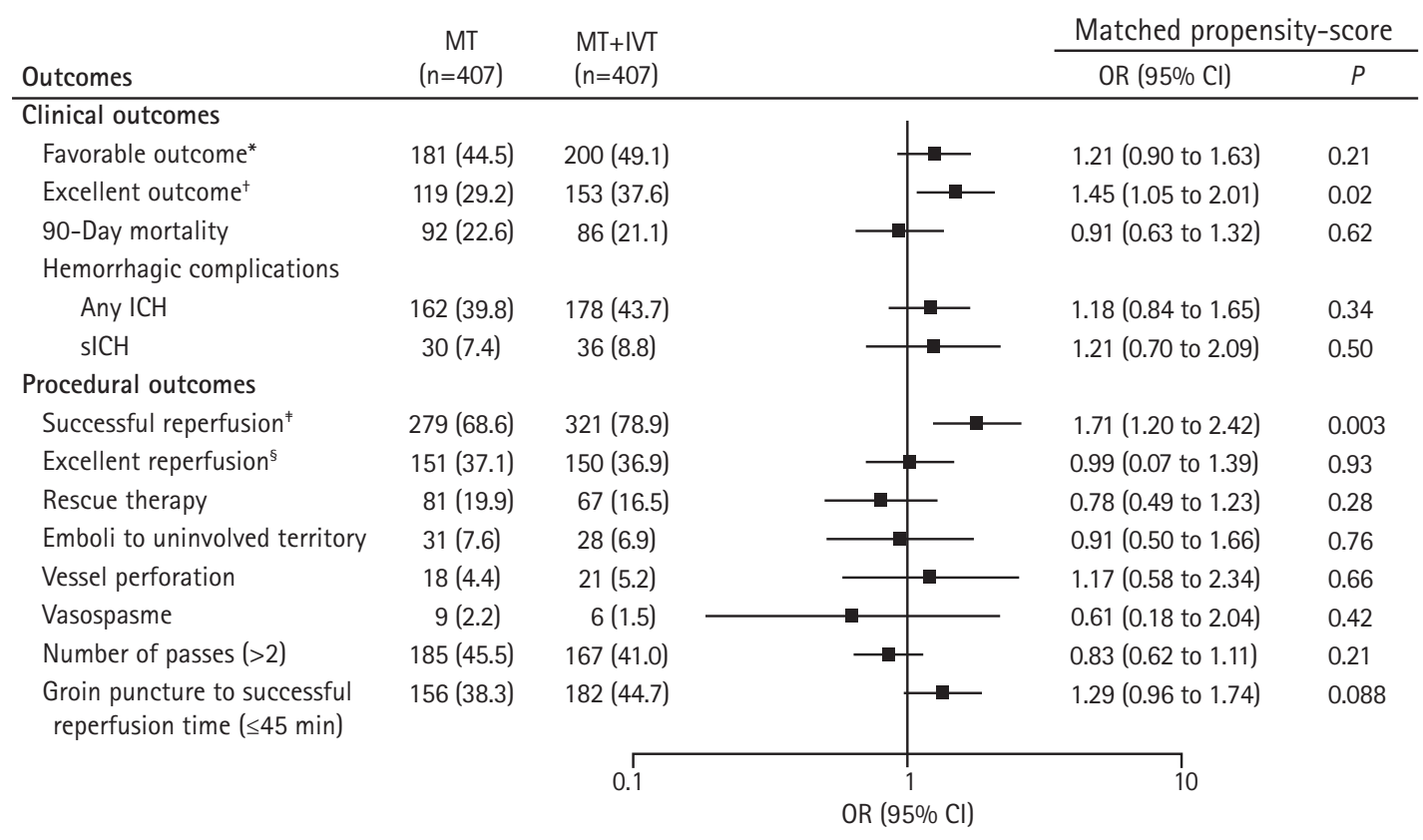

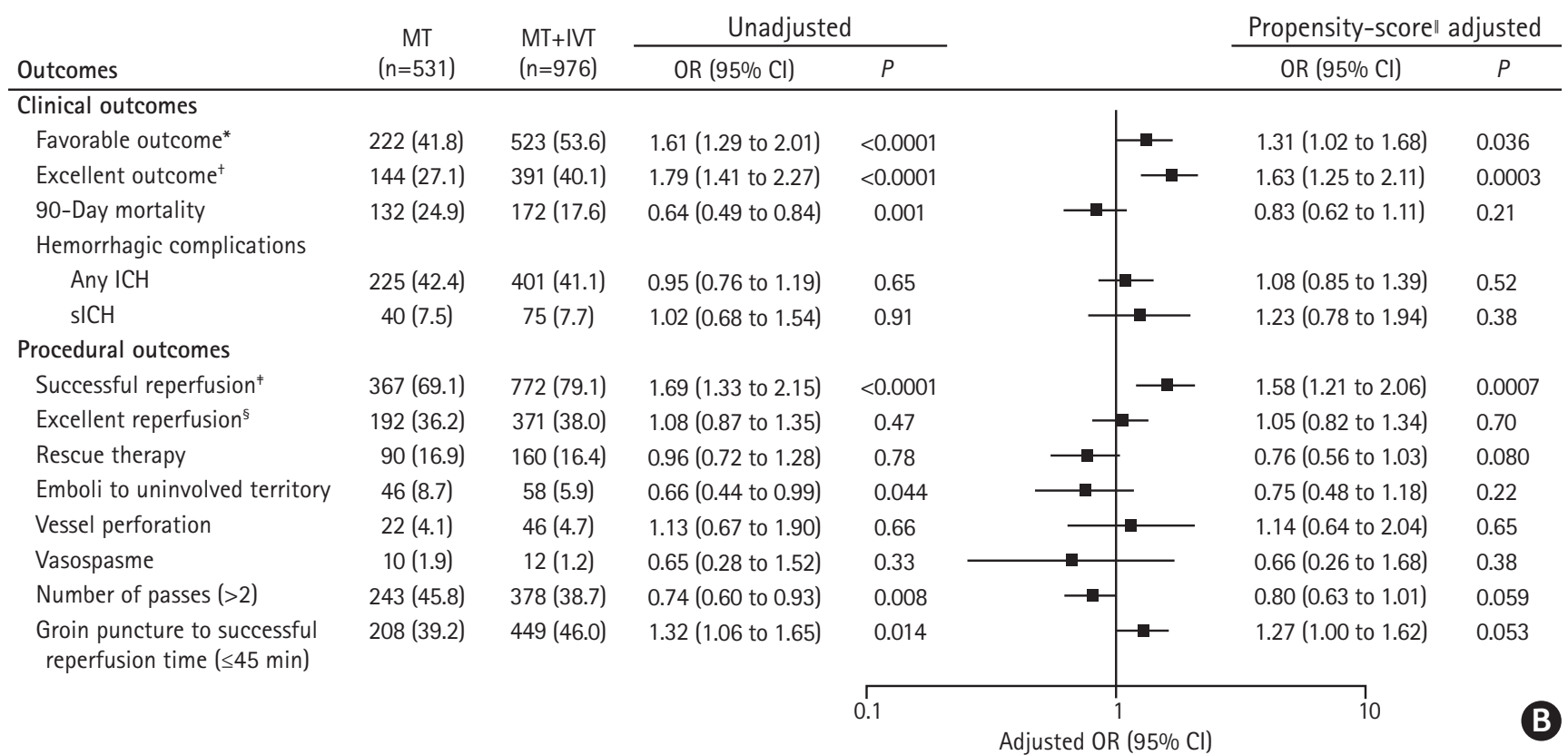

Figure 1. Comparisons in clinical outcomes according to use of intravenous thrombolysis prior to endovascular treatment after (A) and before propensity score-matching (B). Odds ratios (ORs) were calculated after handling missing values for variables included in the propensity score using a multiple imputation procedure. MT, mechanical thrombectomy; IVT, intravenous thrombolysis; $\mathrm{Cl}$, confidence interval; ICH, intracerebral hemorrhage; sICH, symptomatic intracerebral hemorrhage. *Pre-specified primary outcome defined as a 90-day mRS score of 0 to 2, or equal to pre-stroke modified Rankin Scale (mRS) score; ${ }^{+}$Defined as a 90-day mRS of 0 to 1 , or equal to pre-stroke Rankin Score; ${ }^{*}$ Defined as modified Thrombolysis In Cerebral Infarction (mTICl) $2 \mathrm{~b} / 3$; ${ }^{\circledR}$ Defined as mTICl 3 ; " Propensity score was calculated with all parameters in Table 1.

significant in the propensity-score matched-cohort but they are close to significance for the number of passes more than 2 (adjusted $\mathrm{OR}, 0.80 ; 95 \% \mathrm{Cl}, 0.63$ to $1.01 ; P=0.059$ ) and for time from groin puncture to successful reperfusion $\leq 45$ minutes (adjusted $\mathrm{OR}, 1.27 ; 95 \% \mathrm{Cl}, 1.00$ to $1.62 ; P=0.053$ ) in the pro- pensity score-adjusted cohort. In IPTW analyses, the association of IVT+MT with number of passes more than two and time from groin puncture to successful reperfusion $\leq 45$ minutes remained significant (Supplementary Table 3). 


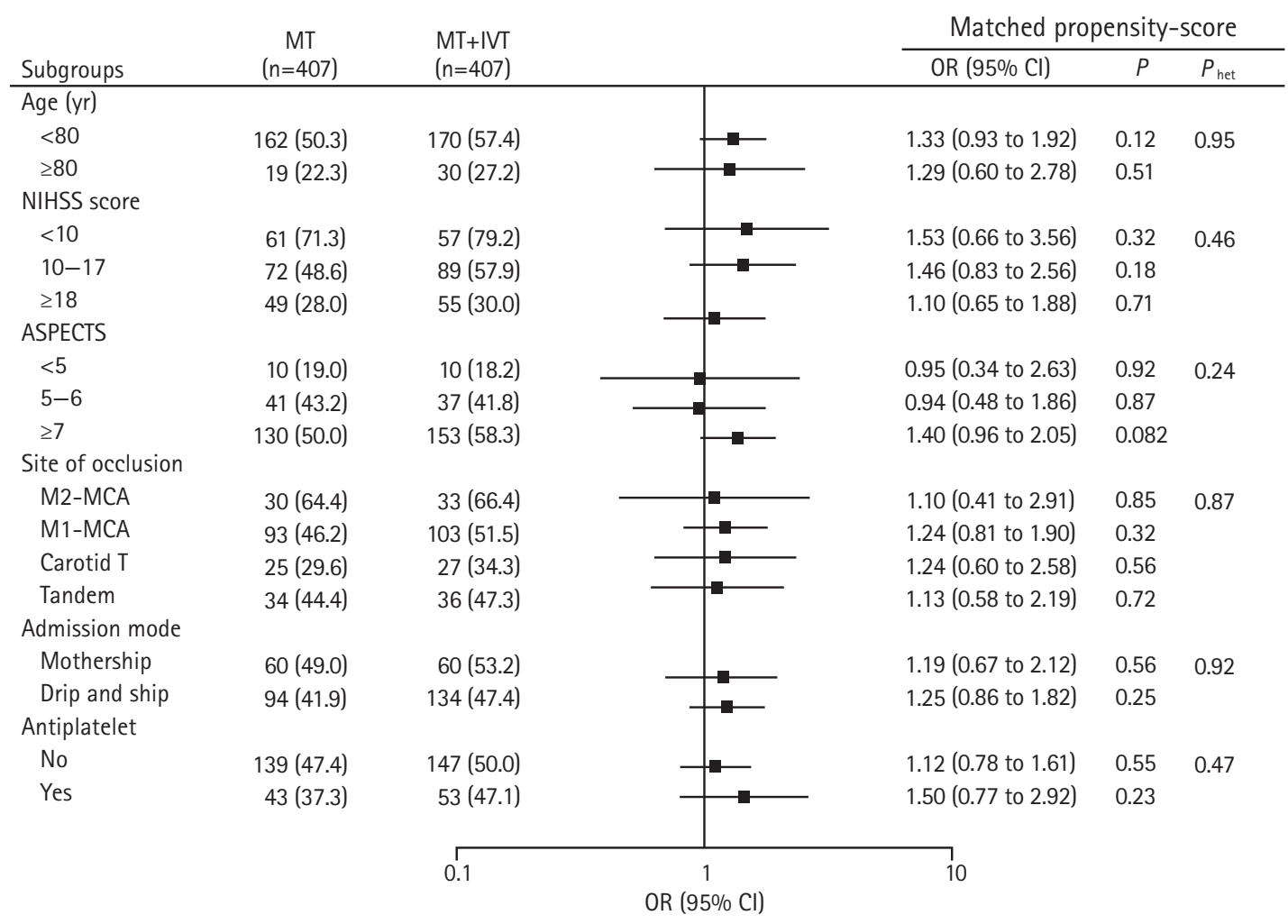

\begin{tabular}{|c|c|c|c|c|c|c|c|c|}
\hline \multirow[b]{2}{*}{ Subgroups } & \multirow{2}{*}{$\underset{(n=531)}{M T}$} & \multirow{2}{*}{$\begin{array}{l}\mathrm{MT}+\mathrm{IVT} \\
(\mathrm{n}=976)\end{array}$} & \multicolumn{2}{|c|}{ Unadjusted } & & \multicolumn{3}{|c|}{ Propensity-score ${ }^{*}$ adjusted } \\
\hline & & & OR $(95 \% \mathrm{Cl})$ & $P$ & & OR $(95 \% \mathrm{Cl})$ & $P$ & $P_{\text {het }}$ \\
\hline \multicolumn{9}{|l|}{ Age (yr) } \\
\hline$<80$ & $194(48.0)$ & $443(61.1)$ & 1.71 (1.32 to 2.21$)$ & $<0.0001$ & $\rightarrow-$ & 1.39 (1.04 to 1.86$)$ & 0.025 & 0.91 \\
\hline$\geq 80$ & $28(22.2)$ & $80(31.9)$ & 1.64 (0.98 to 2.75$)$ & 0.059 & $\longrightarrow$ & 1.35 (0.79 to 2.29$)$ & 0.27 & \\
\hline \multicolumn{9}{|l|}{ NIHSS score } \\
\hline$<10$ & 75 (71.8) & $148(82.9)$ & 1.91 (1.06 to 3.44$)$ & 0.031 & & 1.55 (0.85 to 2.84$)$ & 0.16 & 0.75 \\
\hline $10-17$ & 90 (47.9) & $220(61.3)$ & 1.72 (1.19 to 2.49$)$ & 0.004 & $\longrightarrow$ & 1.44 (0.98 to 2.11$)$ & 0.060 & \\
\hline$\geq 18$ & $57(23.9)$ & $156(35.5)$ & 1.76 (1.21 to 2.56$)$ & 0.003 & $\longrightarrow$ & 1.37 (0.91 to 2.08$)$ & 0.13 & \\
\hline \multicolumn{9}{|l|}{ ASPECTS } \\
\hline$<5$ & $11(15.0)$ & $23(20.0)$ & 1.42 (0.64 to 3.15$)$ & 0.39 & 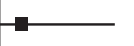 & 1.21 (0.54 to 2.71$)$ & 0.64 & 0.31 \\
\hline $5-6$ & 47 (39.2) & $92(43.6)$ & 1.20 (0.73 to 1.98$)$ & 0.47 & & $1.03(0.62$ to 1.74$)$ & 0.90 & \\
\hline$\geq 7$ & $164(48.8)$ & $409(62.6)$ & 1.76 (1.34 to 2.31$)$ & $<0.0001$ & $\rightarrow$ & 1.48 (1.10 to 2.00$)$ & 0.010 & \\
\hline \multicolumn{9}{|l|}{ Site of occlusion } \\
\hline M2-MCA & $40(61.2)$ & 89 (69.2) & 1.42 (0.76 to 2.69 ) & 0.27 & 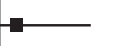 & 1.16 (0.60 to 2.23 ) & 0.66 & 0.75 \\
\hline M1-MCA & $116(43.2)$ & $273(54.8)$ & 1.59 (1.16 to 2.17$)$ & 0.004 &  & 1.30 (0.92 to 1.82$)$ & 0.13 & \\
\hline Carotid T & $30(26.8)$ & 70 (41.3) & $1.92(1.12$ to 3.31$)$ & 0.019 & $\longrightarrow$ & 1.58 (0.90 to 2.76 ) & 0.11 & \\
\hline Tandem & $36(42.6)$ & 91 (50.8) & 1.39 (0.82 to 2.36$)$ & 0.22 & 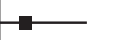 & 1.26 (0.74 to 2.14$)$ & 0.40 & \\
\hline \multicolumn{9}{|l|}{ Admission mode } \\
\hline Mothership & $71(44.4)$ & 179 (59.9) & 1.87 (1.27 to 2.77$)$ & 0.002 & $\longrightarrow$ & 1.44 (0.95 to 2.19 ) & 0.083 & 0.32 \\
\hline Drip and ship & 115 (39.7) & $326(50.3)$ & 1.54 (1.14 to 2.07 ) & 0.005 & 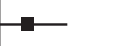 & 1.28 (0.93 to 1.75$)$ & 0.078 & \\
\hline \multicolumn{9}{|l|}{ Antiplatelet } \\
\hline No & $175(44.5)$ & 381 (53.9) & 1.45 (1.12 to 1.88$)$ & 0.004 & $F$ & 1.14 (0.86 to 1.53$)$ & 0.35 & 0.068 \\
\hline Yes & 47 (34.1) & $143(53.0)$ & 2.17 (1.40 to 3.38$)$ & 0.0006 & $\longrightarrow$ & 1.86 (1.18 to 2.91$)$ & 0.007 & \\
\hline
\end{tabular}

Figure 2. Comparisons in favorable outcomes rate according to use of intravenous thrombolysis prior to endovascular treatment after (A) and before propensity score-matching (B) according to key subgroups. Odds ratios (ORs) were calculated after handling missing values for variables included in the propensity score using a multiple imputation procedure. MT, mechanical thrombectomy; IVT, intravenous thrombolysis; $\mathrm{Cl}$, confidence interval; het, heterogeneity; NIHSS, National Institutes of Health Stroke Scale; ASPECTS, Alberta Stroke Program Early CT Score; MCA, middle cerebral artery. *Propensity score was calculated with all parameters in Table 1. 


\section{Subgroup analysis}

When the analysis in primary outcome (favorable) was stratified according to key subgroups, we found no significant heterogeneity in the treatment effect size (Figure 2). In the propensity score-adjusted cohort, we found only a non-significantly better effect of IVT+MT treatment in patients on antiplatelet drugs compared to patients not on antiplatelet drugs at stroke onset ( $P$ for heterogeneity $=0.068$ ); the adjusted OR for IVT+MT was $1.68(95 \% \mathrm{Cl}, 1.06$ to $2.68 ; P=0.029)$ in patients on antiplatelet drugs and $1.20(95 \% \mathrm{Cl}, 0.90$ to $1.61 ; P=0.21)$ in patients not on antiplatelet drugs. In IPTW analyses, we found only a significantly better effect of IVT+MT treatment in patients with ASPECTS $\geq 7(\mathrm{OR}, 1.67 ; 95 \% \mathrm{Cl}, 1.26$ to 2.22$)$ compared to patients with ASPECTS $<5(\mathrm{OR}, 1.02 ; 95 \% \mathrm{Cl}, 0.47$ to 2.24) (Supplementary Table 4).

\section{Discussion}

In the present study, even though IVT+MT didn't prove significantly superior to MT alone in terms of overall better functional ouctome, IVT prior to MT compared to MT alone was associated with higher rates of excellent functional outcome and successful reperfusion. This finding are concordant with the fact that better effects were achieved by IVT+MT in patients with smaller ischemic foci (ASPECTS $\geq 7$ ). There was no significant impact on mortality, and no increased hemorrhagic risk was observed in IVT-treated patients. These findings seem to follow those of an ancillary study within the Enhanced Control of Hypertension and Thrombolysis Stroke Study (ENCHANTED) cohort, in which a tendency toward a better functional outcome was observed in patients who had received a standard dose of intravenous recombining tissue plasminogen activator prior to thrombectomy. ${ }^{19}$

Since December 2014, eight randomized controlled clinical trials have demonstrated the value of MT in addition to IVT for the treatment of AIS patients harboring a large vessel occlusion in the anterior circulation. ${ }^{2,20}$

A majority of patients enrolled in these trials had received IV tPA before MT. As a consequence, European and American recommendations currently advocate the systematic administration of IVT prior to MT in all patients in which IVT is not contraindicated. Conversely, MT alone has proven effective, improving outcomes in patients otherwise ineligible for IVT. ${ }^{6}$

The question of whether MT without prior IVT is better or worse than the combined treatment is now a matter of strong debate. ${ }^{8,20-22}$ Many arguments have been advanced in favour of pretreatment with IVT, including the opportunity of an early reperfusion or even in case of failure of MT; and the possible re-opening of distal occluded branches after MT., ${ }^{1,21}$ Also, it should be recalled that IV tPA allows up to $29 \%$ of early reperfusion visible at the first angiography run, especially in cases of near-occlusion or short thrombi. ${ }^{23}$ Furthermore, prior IVT may enhance the efficacy of MT, with reduced procedure times and higher percentages of successful recanalisation after a single pass., ${ }^{4,24}$ In our study, we noted a significantly lower number of passes required to achieve reperfusion and shorter MT procedural times, as well as higher recanalisation rates for patients who received IVT prior to MT (Figure 2).

On the other hand, IVT may increase thrombus fragility and fragmentation, leading to distal embolic showers while facilitating reperfusion by clot softening, besides the well-known inherent hemorrhagic risk. ${ }^{9,25}$

More importantly, IVT delays endovascular treatment initiation and may result in a worse clinical outcome, especially when administered in the setting of a "drip and ship" protocol. ${ }^{18,26}$ Such a difference has not emerged from our study.

This potential dual nature of IVT outlines a clinical dilemma and is the core of the scientific controversy. ${ }^{11,18}$ The evidence collected up to now brings the debate to a situation of clinical equipoise. Multicenter randomized control trials such as SWIFT DIRECT and MR CLEAN No IV comparing the two treatment approaches are currently ongoing and will hopefully provide definitive information on the efficacy and safety of MT compared with combined IVT+MT.

Limitations to this study are inherent to the non-randomized design of the ETIS registry. The present findings are derived from observational analyses which are subject to well-known limitations. The first is the potential for confounding by measured or unmeasured variables, which cannot be ruled out, even after propensity score matching or adjustment. A second limitation was the presence of missing data in some covariates, including in the propensity score calculation. Although we used multiple imputations to handle missing data as appropriate ${ }_{1}^{27}$ we could not exclude that missing data could introduce a bias in estimates. However, this large real life registry may allow propension score-based analysis and subgroup analysis.

A further bias may exist, since patients not eligible for IVT presented more comorbidities in comparison to eligible patients (c.f., baseline characteristics). Interestingly, the use of IVT in conjunction with MT was associated with a lower occurrence of embolism in new territories, in contrast to observations by other authors. ${ }^{7.26}$ Our findings are on the other hand concordant with the post hoc analysis from the ESCAPE trial ${ }^{28}$ in which IVT was associated with a significant reduction in the occurrence of embolism to a new territory (3.0\% with vs. 9.1\% without; $\mathrm{OR}, 0.32 ; 95 \% \mathrm{Cl}, 0.11$ to 0.96$)$. In addition, we found 
no significant difference in patient outcome according to "mothership" versus "drip and ship" admission mode.

\section{Conclusions}

Patients treated with IVT prior to MT had increased reperfusion rates, reduced MT procedural times and subsequently good functional outcomes. Pending the results of the on-going randomized studies, our findings support a benefit-risk ratio in favor of combined IVT and MT, with better successful reperfusion rates, without increasing embolism in new territories or intracranial hemorrhage rate, in comparison to MT alone.

\section{Supplementary materials}

Supplementary materials related to this article can be found online at https://doi.org/10.5853/jos.2018.01543.

\section{Disclosure}

The authors have no financial conflicts of interest.

\section{Acknowledgments}

We thank Mary Osborne-Pellegrin for help in editing the final draft of the manuscript.

\section{References}

1. Badhiwala JH, Nassiri F, Alhazzani W, Selim MH, Farrokhyar F, Spears J, et al. Endovascular thrombectomy for acute ischemic stroke: a meta-analysis. JAMA 2015;314:1832-1843.

2. Goyal M, Menon BK, van Zwam WH, Dippel DW, Mitchell PJ, Demchuk AM, et al. Endovascular thrombectomy after large-vessel ischaemic stroke: a meta-analysis of individual patient data from five randomised trials. Lancet 2016;387: 1723-1731.

3. Tsivgoulis G, Zand R, Katsanos AH, Turc G, Nolte $\mathrm{CH}_{\text {, Jung }} \mathrm{S}$, et al. Risk of symptomatic intracerebral hemorrhage after intravenous thrombolysis in patients with acute ischemic stroke and high cerebral microbleed burden: a meta-analysis. JAMA Neurol 2016;73:675-683.

4. Powers WJ, Rabinstein AA, Ackerson T, Adeoye OM, Bambakidis NC, Becker K, et al. 2018 Guidelines for the early management of patients with acute ischemic stroke: a guideline for healthcare professionals from the American Heart Association/American Stroke Association. Stroke 2018;49:e46-e110
5. Fiehler J, Cognard C, Gallitelli M, Jansen O, Kobayashi A, Mattle HP, et al. European Recommendations on Organisation of Interventional Care in Acute Stroke (EROICAS). Int J Stroke 2016;11:701-716.

6. Broeg-Morvay A, Mordasini P, Bernasconi C, Bühlmann M, Pult $F_{1}$ Arnold $M$, et al. Direct mechanical intervention versus combined intravenous and mechanical intervention in large artery anterior circulation stroke: a matched-pairs analysis. Stroke 2016;47:1037-1044.

7. Weber $R$, Nordmeyer $H$, Hadisurya J, Heddier $M$, Stauder $M$, Stracke $\mathrm{P}$, et al. Comparison of outcome and interventional complication rate in patients with acute stroke treated with mechanical thrombectomy with and without bridging thrombolysis. J Neurointerv Surg 2017;9:229-233.

8. Tsivgoulis G, Katsanos AH, Mavridis D, Magoufis G, Arthur A, Alexandrov AV. Mechanical thrombectomy improves functional outcomes independent of pretreatment with intravenous thrombolysis. Stroke 2016;47:1661-1664.

9. Tsivgoulis G, Zand R, Katsanos AH, Goyal N, Uchino K, Chang $J$, et al. Safety of intravenous thrombolysis in stroke mimics: prospective 5-year study and comprehensive meta-analysis. Stroke 2015;46:1281-1287.

10. Desilles JP, Consoli A, Redjem H, Coskun O, Ciccio G, Smajda $S$, et al. Successful reperfusion with mechanical thrombectomy is associated with reduced disability and mortality in patients with pretreatment diffusion-weighted imaging: Alberta stroke program early computed tomography score $\leq 6$. Stroke 2017;48:963-969.

11. Campbell BCV, Donnan GA, Davis SM. Optimizing systems of care for endovascular thrombectomy in ischemic stroke: drip and ship versus mothership. Circulation 2017;136:23222324.

12. Dargazanli C, Consoli A, Gory B, Blanc R, Labreuche J, Preda $C_{\text {, et }}$ al. Is reperfusion useful in ischaemic stroke patients presenting with a low national institutes of health stroke scale and a proximal large vessel occlusion of the anterior circulation? Cerebrovasc Dis 2017;43:305-312.

13. Austin PC. An introduction to propensity score methods for reducing the effects of confounding in observational studies. Multivariate Behav Res 2011:46:399-424.

14. Austin PC. A comparison of 12 algorithms for matching on the propensity score. Stat Med 2014;33:1057-1069.

15. Austin PC. Optimal caliper widths for propensity-score matching when estimating differences in means and differences in proportions in observational studies. Pharm Stat 2011;10:150-161.

16. Austin PC. Balance diagnostics for comparing the distribution of baseline covariates between treatment groups in 
propensity-score matched samples. Stat Med 2009;28:30833107.

17. Mattei A. Estimating and using propensity score in presence of missing background data: an application to assess the impact of childbearing on wellbeing. Stat Methods App/ 2009;18:257273.

18. Rubin DB. Multiple Imputation for Nonresponse in Surveys. New York, NY: Wiley, 1987.

19. Kim JS, Kim YJ, Lee KB, Cha JK, Park JM, Hwang Y, et al. Lowversus standard-dose intravenous alteplase in the context of bridging therapy for acute ischemic stroke: a Korean ENCHANTED study. J Stroke 2018;20:131-139.

20. Broderick JP, Palesch YY, Demchuk AM, Yeatts SD, Khatri P, Hill MD, et al. Endovascular therapy after intravenous t-PA versus t-PA alone for stroke. N Engl J Med 2013;368:893-903.

21. Coutinho JM, Liebeskind DS, Slater LA, Nogueira RG, Clark W, Dávalos $A$, et al. Combined intravenous thrombolysis and thrombectomy vs thrombectomy alone for acute ischemic stroke: a pooled analysis of the SWIFT and STAR studies. JAMA Neurol 2017;74:268-274.

22. Tsivgoulis $G$, Alleman J, Katsanos $A H$, Barreto $A D$, Kohrmann $M$, Schellinger PD, et al. Comparative efficacy of different acute reperfusion therapies for acute ischemic stroke: a comprehensive benefit-risk analysis of clinical trials. Brain Behav 2014;4:789-797.

23. Campbell BC, Mitchell PJ, Churilov L, Yassi N, Kleinig TJ, Yan
$B$, et al. Tenecteplase versus alteplase before endovascular thrombectomy (EXTEND-IA TNK): a multicenter, randomized, controlled study. Int J Stroke 2018;13:328-334.

24. Desilles JP, Loyau S, Syvannarath V, Gonzalez-Valcarcel J, Cantier $M$, Louedec $L$, et al. Alteplase reduces downstream microvascular thrombosis and improves the benefit of large artery recanalization in stroke. Stroke 2015;46:3241-3248.

25. Mistry EA, Mistry AM, Nakawah MO, Chitale RV, James RF, Volpi JJ, et al. Mechanical thrombectomy outcomes with and without intravenous thrombolysis in stroke patients: a metaanalysis. Stroke 2017;48:2450-2456.

26. Mokin M, Kass-Hout T, Kass-Hout O, Dumont TM, Kan P, Snyder KV, et al. Intravenous thrombolysis and endovascular therapy for acute ischemic stroke with internal carotid artery occlusion: a systematic review of clinical outcomes. Stroke 2012;43:2362-2368.

27. Cohen J. Statistical Power Analysis for the Behavioral Sciences. 2nd ed. Hillsdale, NJ: Lawrence Erlbaum Associates Publishers, 1988.

28. Ganesh A, Al-Ajlan FS, Sabiq F, Assis Z, Rempel JL, Butcher K, et al. Infarct in a new territory after treatment administration in the ESCAPE randomized controlled trial (endovascular treatment for small core and anterior circulation proximal occlusion with emphasis on minimizing $\mathrm{CT}$ to recanalization times). Stroke 2016;47:2993-2998. 
Supplementary Table 1. Baseline characteristics before imputation

\begin{tabular}{|c|c|c|c|}
\hline \multirow{2}{*}{ Characteristic } & \multicolumn{3}{|c|}{ Before multiple imputation } \\
\hline & MT $(n=531)$ & $\mathrm{MT}+\mathrm{IVT}(\mathrm{n}=976)$ & ASD $(\%)$ \\
\hline Age $(y r)^{*}$ & $67.6 \pm 15.1$ & $67.2 \pm 15.0$ & 2.6 \\
\hline Male sex & 272/531 (51.2) & $530 / 976(54.3)$ & 6.2 \\
\hline Direct admission & 161/531 (30.3) & 299/976 (30.6) & 0.7 \\
\hline \multicolumn{4}{|l|}{ Medical history } \\
\hline Hypertension & $311 / 531(58.6)$ & $507 / 970(52.3)$ & 12.7 \\
\hline Diabetes & $92 / 529(17.4)$ & $154 / 970$ (15.9) & 4.1 \\
\hline Hypercholesterolemia & 155/528 (29.4) & 286/968 (29.5) & 0.4 \\
\hline Current smoking & $132 / 487(27.1)$ & 219/939 (23.3) & 8.7 \\
\hline Antiplatelet use & $134 / 517$ (25.9) & $242 / 879(27.5)$ & 3.6 \\
\hline Anticoagulations & 175/517 (33.8) & $67 / 879(7.6)$ & 68.4 \\
\hline NIHSS score ${ }^{+}$ & $16(11-21.0)$ & $17(11-20)$ & 1.3 \\
\hline ASPECTS ${ }^{\neq}$ & $7(6-8)$ & $7(6-8)$ & 5.2 \\
\hline Pre-stroke rankin $\geq 1$ & $119 / 513(23.2)$ & $111 / 943$ (11.8) & 30.4 \\
\hline Site of occlusion & & & 11.4 \\
\hline M1-MCA & 269/531 (50.7) & 498/973 (51.2) & \\
\hline M2-MCA & $65 / 531(12.2)$ & 128/973 (13.2) & \\
\hline Carotid T & $113 / 531(21.3)$ & 168/973 (17.3) & \\
\hline Tandem & 84/531 (15.8) & 179/973 (18.4) & \\
\hline Favorable collaterals & 160/297 (53.9) & $354 / 600$ (59.0) & 10.4 \\
\hline Stroke etiology & & & 15.1 \\
\hline Large-artery atherosclerosis & $72 / 496(14.5)$ & $163 / 870$ (18.7) & \\
\hline Cardioembolic & $279 / 496$ (56.3) & $428 / 870$ (49.2) & \\
\hline Others & $145 / 496$ (29.2) & $279 / 870(32.1)$ & \\
\hline \multicolumn{4}{|l|}{ EVT characteristics } \\
\hline Onset to groin puncture $(\mathrm{min})^{\S}$ & $247(187-310)$ & $241(200-290)$ & 17.0 \\
\hline Onset to imaging " & $120(85-178)$ & 110 (88-143) & 29.6 \\
\hline Delay imaging-puncture & $108(68-156)$ & 127 (95-158) & 8.1 \\
\hline General anesthesia & 159/522 (30.5) & 211/952 (22.2) & 18.9 \\
\hline First-line strategy & & & 3.5 \\
\hline ADAPT & $235 / 515(45.6)$ & $428 / 941(45.5)$ & \\
\hline Stent retriever & $222 / 515(43.1)$ & $402 / 941$ (42.7) & \\
\hline ADAPT+stent retriever & $34 / 515(6.6)$ & 70/941 (7.4) & \\
\hline Other & $24 / 515(4.7)$ & $41 / 941(4.4)$ & \\
\hline
\end{tabular}

Values are presented as mean \pm standard deviation, number (\%), or median (interquartile range).

MT, mechanical thrombectomy; IVT, intravenous thrombolysis; ASD, absolute standardized difference; NIHSS, National Institutes of Health Stroke Scale; ASPECTS, Alberta Stroke Program Early CT Score; MCA, middle cerebral artery; EVT, endovascular treatment; ADAPT, a direct aspiration first pass technique. Number of missing values: ${ }^{*} 0 / 1,507 ;{ }^{\dagger} 15 / 1,507 ;{ }^{\dagger} 45 / 1,507 ;{ }^{\text {s. }} 11, \uparrow^{9} 346 / 1,507$. 
Supplementary Table 2. Standardized differences in baseline characteristics in propensity score adjusted- and IPTW-cohorts

\begin{tabular}{|c|c|c|}
\hline Baseline characteristic & Propensity-score adjusted cohort & IPTW cohort \\
\hline Age & 1.6 & 1.5 \\
\hline Male sex & 0.5 & 0.8 \\
\hline Direct admission & 0.04 & 4.0 \\
\hline \multicolumn{3}{|l|}{ Medical history } \\
\hline Hypertension & 0.3 & 0.2 \\
\hline Diabetes & 0.6 & 0.5 \\
\hline Hypercholesterolemia & 0.5 & 0.6 \\
\hline Current smoking & 1.3 & 0.5 \\
\hline Antiplatelet drugs & 0.7 & 0.9 \\
\hline Anticoagulations & 0.2 & 0.9 \\
\hline NIHSS score & 0.7 & 1.1 \\
\hline ASPECTS & 1.2 & 0.0 \\
\hline Pre-stroke rankin $\geq 1$ & 1.1 & 0.7 \\
\hline \multicolumn{3}{|l|}{ Site of occlusion } \\
\hline M1-MCA & 0.5 & 0.8 \\
\hline M2-MCA & 0.2 & 0.6 \\
\hline Carotid T & 0.2 & 0.1 \\
\hline Tandem & 0.3 & 0.6 \\
\hline Favorable collaterals & 1.0 & 0.2 \\
\hline \multicolumn{3}{|l|}{ Stroke etiology } \\
\hline Large-artery atherosclerosis & 0.2 & 0.6 \\
\hline Cardioembolic & 1.4 & 1.3 \\
\hline Others & 1.4 & 1.9 \\
\hline \multicolumn{3}{|l|}{ EVT characteristics } \\
\hline Onset to groin puncture & 1.2 & 2.0 \\
\hline Onset to imaging & 1.8 & 1.6 \\
\hline Delay imaging-puncture & 0.4 & 4.0 \\
\hline General anesthesia & 1.5 & 1.1 \\
\hline \multicolumn{3}{|l|}{ First-line strategy } \\
\hline ADAPT & 0.1 & 2.6 \\
\hline Stent retriever & 0.4 & 2.9 \\
\hline ADAPT+stent retriever & 0.5 & 0.07 \\
\hline Other & 0.5 & 0.6 \\
\hline
\end{tabular}

Values are presented as absolute standardized differences, expressed as \%. Values were calculated after handing missing data using multiple imputation procedure.

IPTW, inverse probability of treatment weighting; NIHSS, National Institutes of Health Stroke Scale; ASPECTS, Alberta Stroke Program Early CT Score; MCA, middle cerebral artery; EVT, endovascular treatment; ADAPT, a direct aspiration first pass technique. 
Supplementary Table 3. Comparisons in clinical outcomes according to use of intravenous thrombolysis prior to endovascular treatment using the stabilized inverse propensity score of MT+IVT as weight into logistic regression model

\begin{tabular}{|c|c|c|}
\hline Outcomes & OR $(95 \% \mathrm{Cl})$ & $P$ \\
\hline \multicolumn{3}{|l|}{ Clinical outcomes } \\
\hline Favorable outcome & $1.37(1.09-1.73)$ & 0.008 \\
\hline Excellent outcome & $1.71(1.33-2.21)$ & $<0.001$ \\
\hline 90-Day mortality & $0.78(0.60-1.02)$ & 0.065 \\
\hline \multicolumn{3}{|l|}{ Hemorrhagic complications } \\
\hline Any ICH & $1.07(0.85-1.36)$ & 0.550 \\
\hline $\mathrm{sICH}$ & $1.19(0.77-1.82)$ & 0.430 \\
\hline \multicolumn{3}{|l|}{ Procedural outcomes } \\
\hline Successful reperfusion & $1.56(1.22-1.99)$ & $<0.001$ \\
\hline Excellent reperfusion & $1.09(0.87-1.36)$ & 0.440 \\
\hline Rescue therapy & $0.80(0.61-1.04)$ & 0.100 \\
\hline Emboli uninvolved territory & $0.69(0.45-1.04)$ & 0.080 \\
\hline Vessel perfusion & $1.05(0.64-1.73)$ & 0.850 \\
\hline Vasospasme & $0.57(0.25-1.32)$ & 0.190 \\
\hline Number of passes (>2) & $0.79(0.64-0.99)$ & 0.037 \\
\hline Groin puncture to successful reperfusion time (45 min) & $1.31(1.05-1.63)$ & 0.017 \\
\hline
\end{tabular}

IMT, mechanical thrombectomy; IVT, intravenous thrombolysis; $\mathrm{OR}$, odds ratio; $\mathrm{Cl}$, confidence interval; ICH, intracerebral hemorrhage; sICH, symptomatic intracerebral hemorrhage. 
Supplementary Table 4. Comparisons in favorable outcomes rate according to use of intravenous thrombolysis prior to endovascular treatment in key subgroups using the stabilized Inverse propensity score as weight into logistic regression model

\begin{tabular}{|c|c|c|c|}
\hline Subgroup & OR (95\% Cl) & $P$ & $P_{\text {het }}$ \\
\hline Age (yr) & & & 0.820 \\
\hline$<80$ & $1.45(1.11-1.90)$ & 0.007 & \\
\hline$\geq 80$ & $1.55(0.91-2.64)$ & 0.100 & \\
\hline NIHSS score & & & 0.480 \\
\hline$<10$ & $1.56(0.86-2.83)$ & 0.140 & \\
\hline $10-17$ & $1.70(1.18-2.46)$ & 0.005 & \\
\hline$\geq 18$ & 1.31 (0.89-1.94) & 0.170 & \\
\hline ASPECTS & & & 0.041 \\
\hline$<5$ & $1.02(0.47-2.24)$ & 0.950 & \\
\hline $5-6$ & $1.92(0.53-1.56)$ & 0.750 & \\
\hline$\geq 7$ & $1.67(1.26-2.22)$ & $<0.001$ & \\
\hline Site of occlusion & & & 0.580 \\
\hline M2-MCA & $1.50(1.08-2.08)$ & 0.016 & \\
\hline M1-MCA & $1.12(0.58-2.18)$ & 0.730 & \\
\hline Carotid T & $1.45(0.81-2.57)$ & 0.210 & \\
\hline Tandem & $1.24(0.74-2.08)$ & 0.410 & \\
\hline Admission mode & & & 0.500 \\
\hline Mothership & $1.47(0.97-2.24)$ & 0.071 & \\
\hline Drip and ship & $1.34(0.99-1.81)$ & 0.058 & \\
\hline Antiplatelet use & & & 0.160 \\
\hline No & $1.25(0.96-1.63)$ & 0.100 & \\
\hline Yes & $1.79(1.15-2.76)$ & 0.009 & \\
\hline
\end{tabular}

$\mathrm{OR}$, odds ratio; $\mathrm{Cl}$, confidence interval; het, heterogeneity; NIHSS, National Institutes of Health Stroke Scale; ASPECTS, Alberta Stroke Program Early CT Score; $M C A$, middle cerebral artery. 


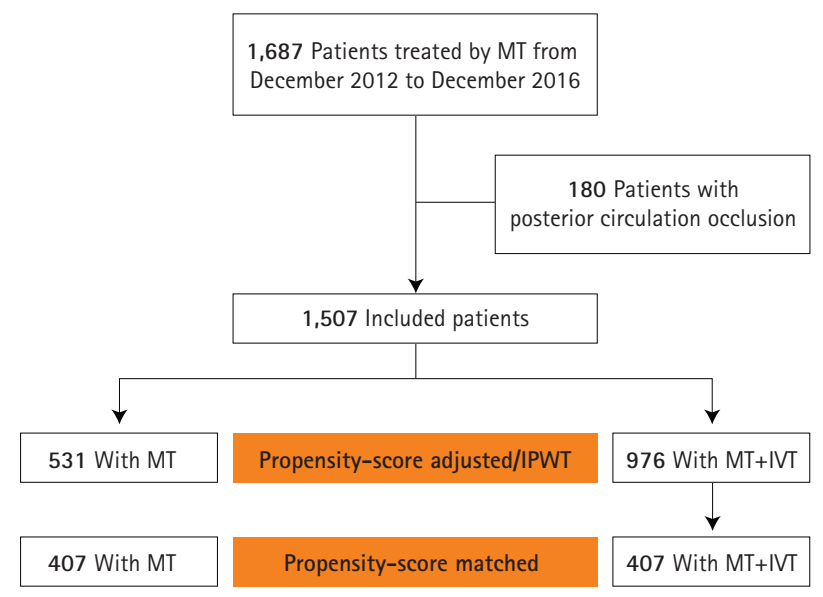

Supplementary Figure 1. Study flow chart. MT, mechanical thrombectomy; IPWT, inverse probability of treatment weighting; IVT, intravenous thrombolysis. 


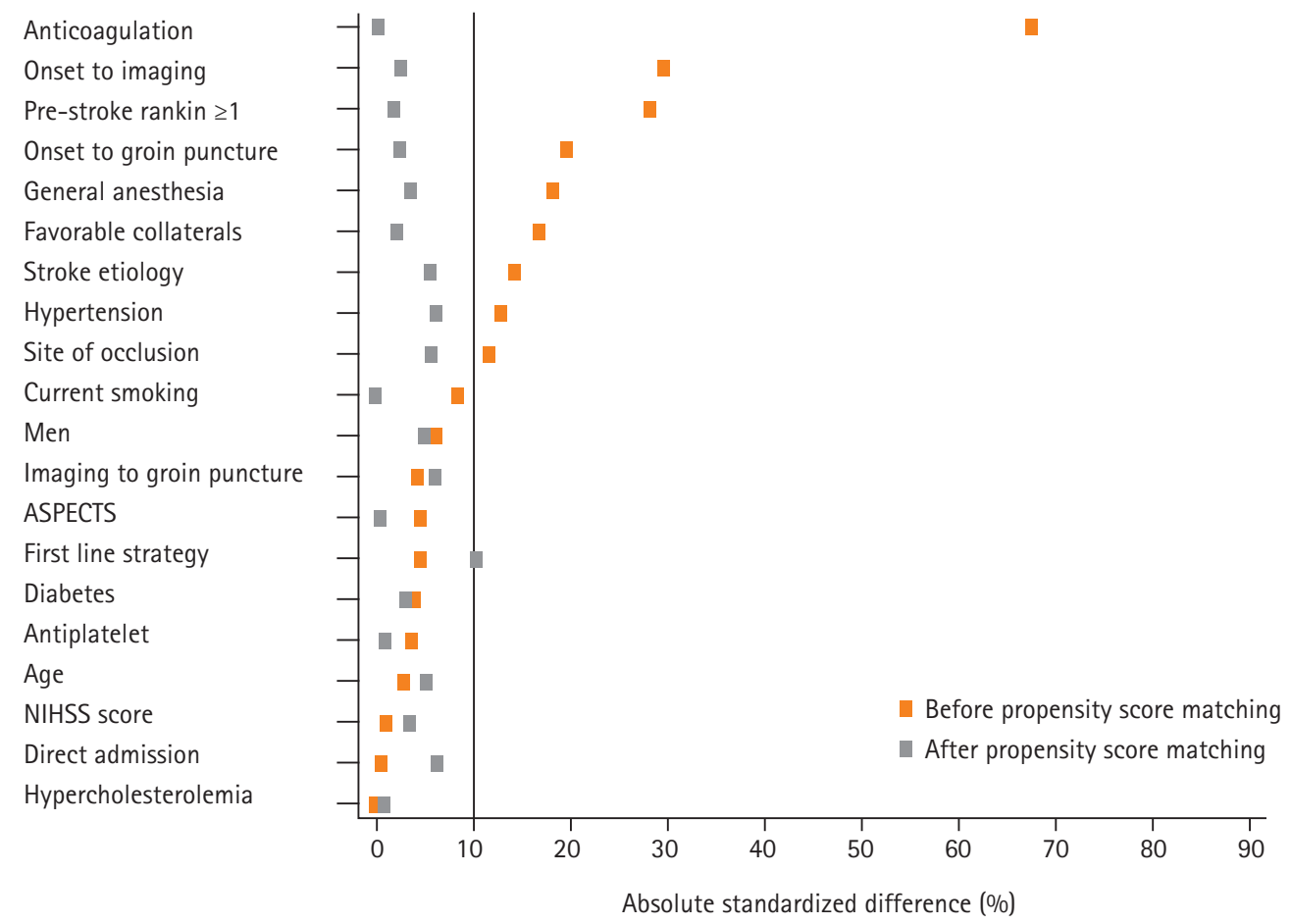

Supplementary Figure 2. Absolute standardized differences between mechanical thrombectomy (MT)+intravenous thrombolysis (IVT) versus MT treatment before and after propensity score matching. ASPECTS, Alberta Stroke Program Early CT Score; NIHSS, National Institutes of Health Stroke Scale. 